\title{
Dynamic Registration Method with Balancing for Prognostic Factors in Observational Studies
}

\author{
Masahiro Sugihara ${ }^{1 *}$, Satoshi Morita ${ }^{2}$, Naoki Yamanouchi ${ }^{1}$, Shinya Sakai ${ }^{3}$, Noriaki Ohba $^{3}$, Wataru Ichikawa ${ }^{4}$ and Yasuo Ohashi $^{5}$ \\ ${ }^{1}$ Clinical Data and Biostatistics Department, R\&D Division, Daiichi Sankyo Co., Ltd., Tokyo, Japan \\ ${ }^{2}$ Department of Biostatistics and Epidemiology, Yokohama City University Graduate School of Medicine and Medical Center, Yokohama, Japan \\ ${ }^{3}$ Statistics Analysis Division 1, Biometrics Department, EPS Co., Ltd., Tokyo, Japan \\ ${ }^{4}$ Department of Clinical Oncology, National Defense Medical College Hospital, Saitama, Japan \\ ${ }^{5}$ Department of Biostatistics/Epidemiology and Preventive Health Sciences, University of Tokyo, Tokyo, Japan
}

\begin{abstract}
Randomized controlled trials are the most scientifically informative studies for evaluating treatment effects. However, we need to conduct observational studies to evaluate unallocatable factors such as genotype, preference, or lifestyle. In observational studies, subject characteristics among the comparison groups might be imbalanced due to non-random allocation. We proposed a dynamic registration method to improve comparability among comparison groups with no allocation. The dynamic registration method is a registration method based on the minimization method, which decides whether or not to register a subject based on the background information of subjects already recruited and the new subject. Simulation studies were conducted to examine the performance of this method in improving comparability among comparison groups. Simulation studies showed that the dynamic registration method improves the comparability among comparison groups. The dynamic registration method can be used to enhance the quality of observational studies for unallocatable factors.
\end{abstract}

Keywords: Dynamic registration; Minimization method; Observational study; Simulation studies

\section{Introduction}

To conduct clinical trials ethically and scientifically, we need to consider various issues at the time of protocol planning. One of the most important elements of the design is the method of treatment allocation. Random allocation of treatments is conducted to evaluate the treatment effect in the most optimal way. However, random allocation has a risk of imbalancing important prognostic factors between the treatment groups, particularly in smaller trials. In clinical trials, imbalances in important prognostic factors degrade the quality of the clinical trial and reduce the statistical efficiency even if the imbalanced factors are adjusted in the statistical analysis [1]. In view of these considerations, various allocation methods have been proposed to avoid chance imbalances [1]. In particular, the methods proposed by Taves [2] and by Pocock and Simon [3], and their modifications are widely known as the minimization method and frequently used in clinical trials. The minimization method can be classified as a dynamic allocation method, as the allocation depends on the prognostic factors of subjects already recruited. The minimization method has been recommended as an effective method for treatment allocation in randomized trials $[4,5]$.

Randomized Controlled Trials (RCTs) are the most scientifically informative studies in the evaluation of treatment effects. However, if one aims to compare patient groups with respect to unallocatable factors such as genotype, preference, and lifestyle, randomization cannot be used. In such cases, since conducting RCTs is difficult, observational studies without random allocation are often conducted.

Recently, a number of genetic polymorphisms have been reported to affect pharmacokinetics and pharmacodynamics of drugs. This field in pharmacology, pharmacogenomics, is rapidly developing, and its outcomes, as sensitive genetic biomarkers for drug safety and efficacy, have been already applied to development and proper usage of drugs. An anticancer drug irinotecan (CPT-11) is metabolized to form active SN-38, which is further conjugated and detoxified by UDPglucuronosyltransferase (UGT) 1A1 enzyme. Genetic polymorphisms of the UGT1A1 would affect an interindividual variation of the toxicity by CPT-11 via the alternation of bioavailability of SN-38 [6,7]. Since concerns have been expressed about severe toxicity, such as diarrhea and neutropenia, for treatment with CPT-11, we planned a prospective observational study to investigate whether a patient with the variant UGT1A1 genotypes would be at higher risk for severe toxicity by CPT-11 in Japanese cancer patients. In this observational study, the frequency of the severe toxicity will be compared among the UGT1A1 genotype groups treated with CPT-11-containing regimens.

RCTs generally evaluate efficacy rather than effectiveness, as there are many restrictions that limit generalizability under restricted conditions. On the other hand, observational studies can evaluate effectiveness under the conditions of real clinical practice [8]. In observational studies, however, unequal distribution of prognostic factors among compared groups causes confounding bias. Although evaluation of the compared factors in observational studies requires adjustment for confounding factors through statistical analyses, if the distributions of the prognostic factors greatly differ among comparison groups, this adjustment is difficult. Methods to adjust for confounding factors have included stratification, regression models such as Cox proportional hazards model, and propensity score methods [9]. However, when the distributions of the prognostic factors hardly overlap among compared groups, the results from statistical analyses should be interpreted carefully [10]. Therefore, even in observational studies, procedure to improve the comparability among comparison

*Corresponding author: Masahiro Sugihara, Clinical Data and Biostatistics Department, R\&D Division, Daiichi Sankyo Co., Ltd., 1-2-58, Hiromachi, Shinagawa-ku, Tokyo 140-8710, Japan, Tel: +81 3-5740-3422; Fax: +81 3-57403607; E-mail: sugihara.masahiro.e5@daiichisankyo.co.jp

Received March 13, 2012; Accepted May 18, 2012; Published May 19, 2012

Citation: Sugihara M, Morita S, Yamanouchi N, Sakai S, Ohba N, et al. (2012) Dynamic Registration Method with Balancing for Prognostic Factors in Observational Studies. J Biomet Biostat S7:012. doi:10.4172/2155-6180.S7-012

Copyright: (c) 2012 Sugihara M, et al. This is an open-access article distributed under the terms of the Creative Commons Attribution License, which permits unrestricted use, distribution, and reproduction in any medium, provided the original author and source are credited. 
groups as much as possible before starting the study might be important and enhance the quality of the study. The matched casecontrol approach is considered as a method for this purpose. However, especially in the case where the number of controls is large relative to the number of cases, this approach requires large resources and costs since this approach needs follow up of all registered subjects until matching pairs are formed.

In this paper, we propose a dynamic registration method which dynamically judges subject registration using the minimization method to reduce resources and costs in conjunction with improvement in comparability for prognostic variables between two groups in the observational studies. We examined the performance of the dynamic registration method for improvement of comparability between two groups through simulation studies.

\section{Methods}

\section{Proposed dynamic registration}

The proposed dynamic allocation method is a prospective registration method which does not register a new subject if it would be difficult to maintain the balance in prognostic factors among groups consisting of unallocatable factors such as subject preferences, habits, and genes if the subject were registered. Note that subjects who are not registered are put in a tentative registration pool as candidates for registration. To apply the dynamic registration method, first, we need to decide the prognostic factors related to the outcome before starting the study. Next, we set the registration probabilities so that the best possible balance was obtained between the comparison groups based on prognostic-factor information of subjects already recruited and a candidate for registration. The registration probability is the probability of registration given for the candidate. The registration probability will be high if registration of the subject would improve the balance in prognostic factors between groups. In contrast, the registration probability will be low and registration of the subject will be difficult if it would adversely affect the balance. The registration procedure is shown in Figure 1.

\section{Procedure of dynamic registration}

The minimization method used in randomized controlled trials was independently proposed by Taves [2] and Pocock and Simon [3], but the method proposed by Taves is often used due to its practical convenience [4]. The dynamic registration method proposed in this study was developed based on Taves' minimization method from

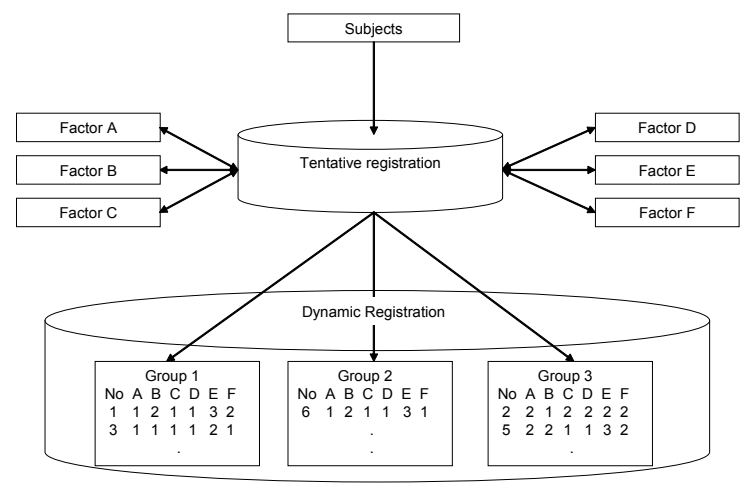

Figure 1: Flow chart of dynamic registration a practical viewpoint. We will explain the procedure of the dynamic registration method based on examples (Table 1) presented by Scott et al. [4].

As shown in Table 1, a total of 16 subjects, 8 in each group, have already been registered in this example. A $17^{\text {th }}$ subject (male, aged 38 and with a high risk factor) has been tentatively registered as a candidate for registration. Whether or not this subject will be registered is decided based on whether the overall balance in prognostic factors can be maintained. The balance between groups is evaluated by comparing the total values of the levels of prognostic factors that correspond to the background of the candidate for registration between groups. If the total becomes nearly equal between groups, it signifies that the overall balance between groups will improve. As shown in Table 1, in this example the $17^{\text {th }}$ subject will be registered as it will improve the overall balance in prognostic factors between groups.

\section{Measures for balance between comparison groups}

Let $N_{k}$, and $N$ be the planned number of subjects for group $k$ ( $k=1$, 2 ) and the total number of subjects in all groups. Let $n_{k}$ and $n$ be the number of subjects in group $k$, immediately before a new subject is tentatively registered and the number of subjects in all groups is totaled. Then, when the number of subjects with level $j\left(j=1,2, \ldots, Q_{i}\right)$ of factor $i(i=1,2, \ldots, P)$ in group $k$ is expressed as $n_{i j k}$, the proportion of level $j$ of factor $i$ in group $k$ becomes $n_{i j k} / n_{k}$. The balance of the distribution of factors between groups is evaluated by the difference in the proportion $n_{i j k} / n_{k}$ for all $i$ and $j$ between groups.

We consider $S_{k}=\sum_{i, j=r_{i}} n_{i j k}$, which is the total number of subjects corresponding to the same level of each factor as a candidate for registration for all factors, as a measure to evaluate the imbalance in the distribution of factors between groups. Here, $r_{i}$ is the level of factor $i$ of the candidate for registration. When the candidate belongs to group $k$, a balance in the distribution of factors might be maintained between group $k_{s}$ and group $k$ within a certain range by registering this candidate in the case of $S_{k_{s}} \leq \frac{N_{k_{s}}}{N_{k}} \cdot S_{k}$. When the planned number of subjects is the same between groups, the condition is $S_{k_{s}} \leq S_{k}$.

\section{Decision of subject registration}

Next, we set the registration probability of a candidate for registration based on each group's $S_{k}(k=1,2)$. We consider group

\begin{tabular}{|l|l|l|}
\hline Prognostic factor & Group 1 & Group 2 \\
\hline Sex & & \\
\hline Male & 3 & 5 \\
\hline Female & 5 & 3 \\
\hline Age band & & \\
\hline $21-30$ & 4 & 4 \\
\hline $31-40$ & 2 & 3 \\
\hline $41-50$ & 2 & 1 \\
\hline Risk factor & & \\
\hline High & 4 & 5 \\
\hline Low & 4 & 3 \\
\hline
\end{tabular}

If the 17th subject has factors Male, 31-40, High in Group 1:

Total in group $1,3+2+4=9$.

Total in group $2,5+3+5=13$.

17 th subject is registered because $9 \leq 13$

Table 1: An example of how the dynamic registration works in a setting of an observational study. 


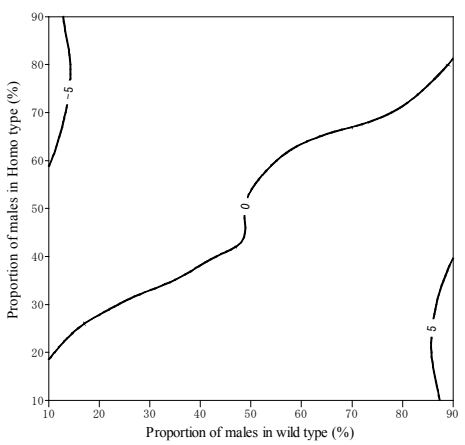

a-1) Number of subjects: 200 Proportion of wild-type subjects: $85 \%$

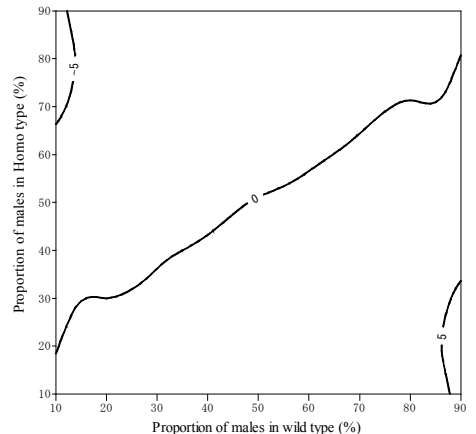

b-1) Number of subjects: 1500 Proportion of wild-type subjects: $85 \%$

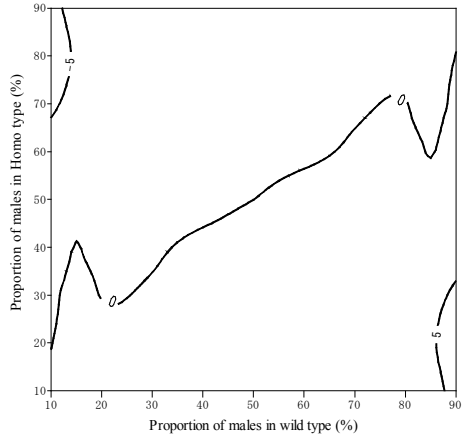

c-1) Number of subjects: 4000

Proportion of wild-type subjects: $85 \%$

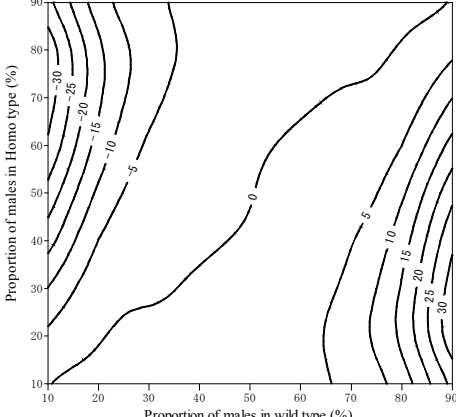

a-2) Number of subjects: 200

Proportion of wild-type subjects: $65 \%$

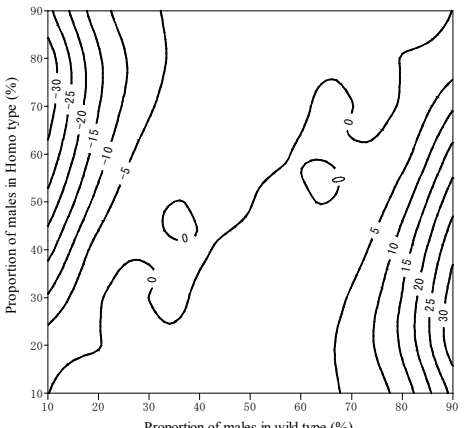

b-2) Number of subjects: 1500

Proportion of wild-type subjects: $65 \%$

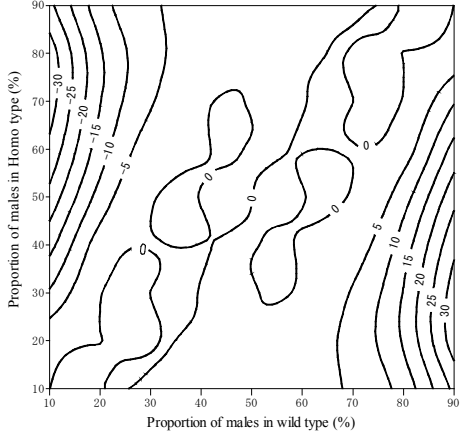

c-2) Number of subjects: 4000

Proportion of wild-type subjects: $65 \%$

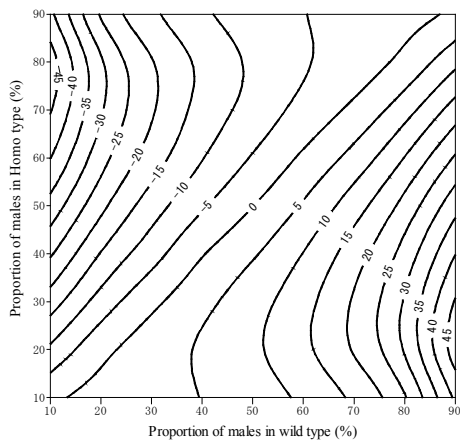

a-3) Number of subjects: 200

Proportion of wild-type subjects: $50 \%$

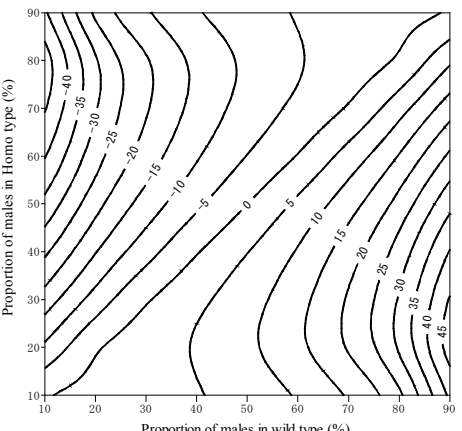

b-3) Number of subjects: 1500

Proportion of wild-type subjects: $50 \%$

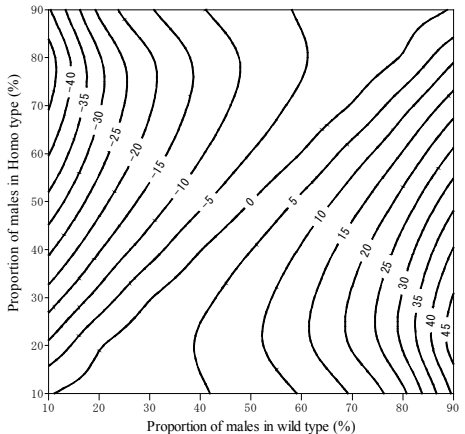

c-3) Number of subjects: 4000

Proportion of wild-type subjects: $50 \%$

Figure 2: Contour line plots of mean of the proportion difference in wild-type and homo-type males in the simulations, with the probability (\%) of a male of the wild type on the $x$-axis and the probability (\%) of a male of the homo type on the $y$-axis.

$k=2$ as a reference group without the dynamic registration $(100 \%$ registration) so as not to unnecessarily increase the number of subjects not registered. The logic for registration of a candidate in group 1 is described as follows.

If $a_{1} S_{1} \leq \frac{N_{1}}{N_{2}} S_{2}$ then $P$ registration of subject in group 1$\}=p_{1}$
Else if $a_{2} S_{1} \leq \frac{N_{1}}{N_{2}} S_{2}$ then $P\{$ registration of subject in group 1$\}=p_{2}$ Else if $a_{L-1} S_{1} \leq \frac{N_{1}}{N_{2}} S_{2}$ then $P\{$ registration of subject in group 1$\}=$ $p_{L}^{-1}$

Else then $P\{$ registration of subject in group 1$\}=p_{L}$ 
Citation: Sugihara M, Morita S, Yamanouchi N, Sakai S, Ohba N, et al. (2012) Dynamic Registration Method with Balancing for Prognostic Factors in Observational Studies. J Biomet Biostat S7:012. doi:10.4172/2155-6180.S7-012

Where $a_{l}(l=1,2, \ldots, L-1)$ is a coefficient that expresses the degree of balance between groups and $p_{l}(l=1,2, \ldots, L)$ is the registration probability, which is decided arbitrarily by consulting with a medical adviser, etc. If $L=4$, for example, $p_{1}=1, p_{2}=0.8, p_{3}=0.5, p_{4}=0, a_{1}=1$, $a_{2}=0.9$, and $a_{3}=0.8$. Like the minimization method proposed by Taves, one option is to not set a registration probability, i.e., register $100 \%$ of subjects if $S_{k_{s}} \leq \frac{N_{k_{s}}}{N_{k}} \cdot S_{k}$ but not register otherwise.

\section{Simulation studies}

We conducted Monte Carlo simulations to evaluate the performance of the proposed method. In the simulations, we considered genotype (wild type, homo type) as a comparison factor. The sole prognostic factor was sex, to make the simulation simple, and the probability of an individual being male in each genotype was set from $10 \%$ to $90 \%$ at $10 \%$ intervals. We simulated three planned sample sizes, 200 (100 subjects per group), 1,500 (750 subjects per group), and 4,000 (2,000 subjects

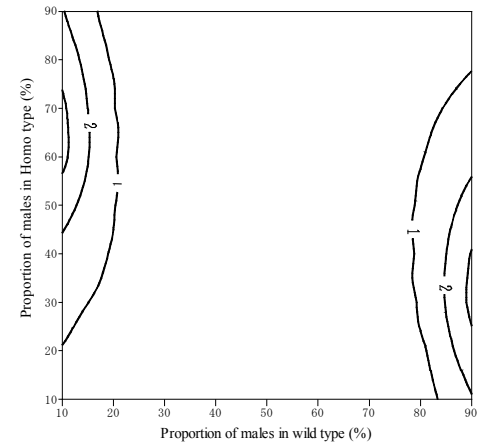

a-1) Number of subjects: 200

Proportion of wild-type subjects: $85 \%$

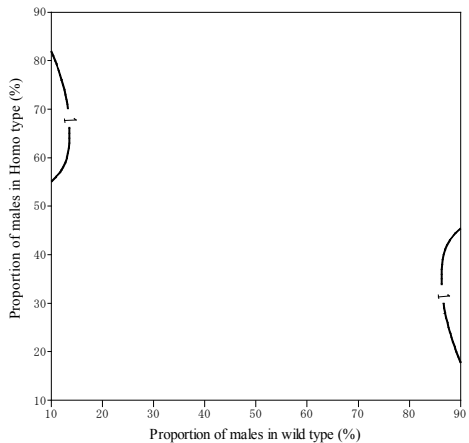

b-1) Number of subjects: 1500 Proportion of wild-type subjects: $85 \%$

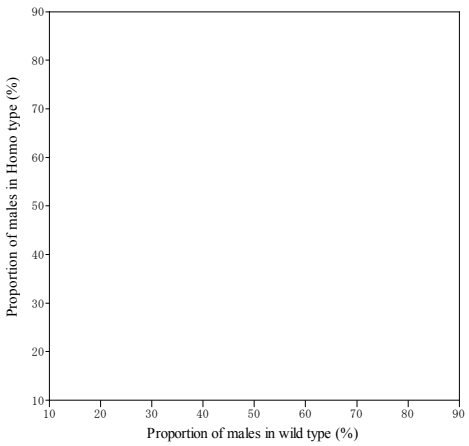

c-1) Number of subjects: 4000

Proportion of wild-type subjects: $85 \%$

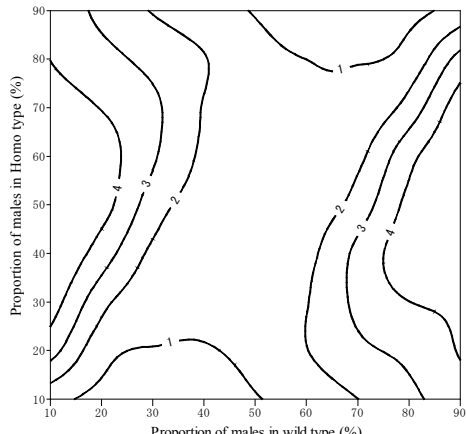

a-2) Number of subjects: 200

Proportion of wild-type subjects: $65 \%$

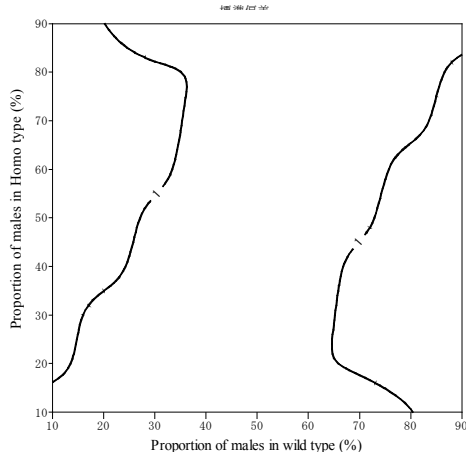

b-2) Number of subjects: 1500

Proportion of wild-type subjects: $65 \%$

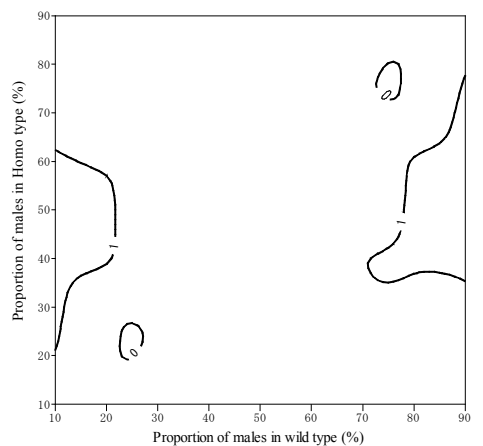

c-2) Number of subjects: 4000

Proportion of wild-type subjects: $65 \%$

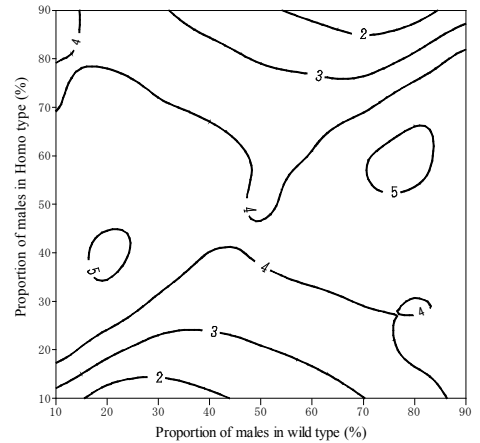

a-3) Number of subjects: 200

Proportion of wild-type subjects: $50 \%$

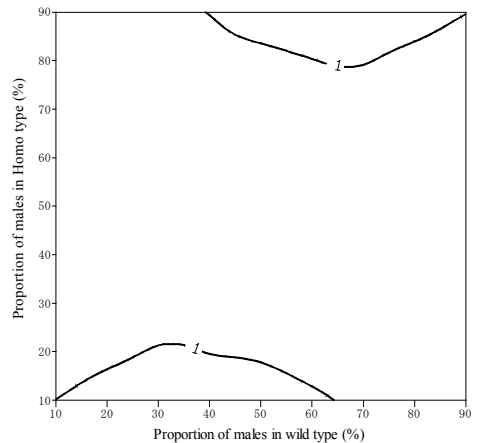

b-3) Number of subjects: 1500

Proportion of wild-type subjects: $50 \%$

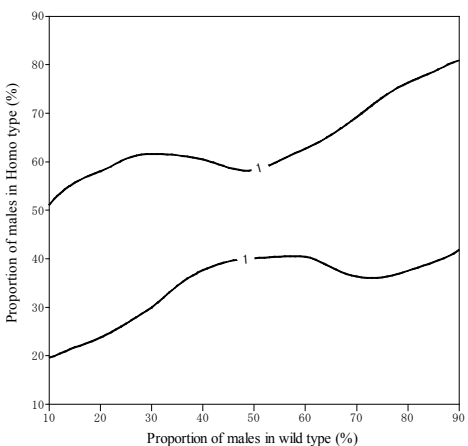

c-3) Number of subjects: 4000

Proportion of wild-type subjects: 50\%

Figure 3: Contour line plots of standard deviation of the proportion difference in wild-type and homo-type males in the simulations, with the probability (\%) of a male of the wild type on the $x$-axis and the probability (\%) of a male of the homo type on the $y$-axis. 
per group), and three proportions of a candidate for registration being wild type in the target population, $85 \%, 65 \%$, and $50 \%(15 \%, 35 \%$, and $50 \%$, respectively, for homo type). The dynamic registration was applied only to the wild type, while $100 \%$ of homo type subjects were registered. Whether or not a wild-type candidate for registration would be registered was decided according to the following logic.

If $0.98 S_{\text {Wild }} \leq S_{\text {Номо }}$ then $P\{$ registration of subject in wild type $\}=p_{1}$

Else if $0.95 S_{\text {Wild }} \leq S_{\text {Homo }}$ then $P$ \{registration of subject in wild type $\}=$ $p_{2}$

Else if $0.90 S_{\text {Wild }} \leq S_{\text {Homo }}$ then $P\{$ registration of subject in wild type $\}=$ $p_{3}$

Else $P$ \{registration of subject in wild type $\}=p_{4}$,

Where $S_{\text {Wild }}$ and $S_{\text {Homo }}$ are the number of subjects of the wild type and homo type with the same sex as that of a candidate for registration, respectively. The following three registration probabilities were set.

$\begin{array}{lllll} & \left\{p_{1},\right. & p_{2}, & p_{3}, & \left.p_{4}\right\} \\ \text { Pattern 1 } & \{1.0, & 0.8, & 0.5, & 0.0\} \\ \text { Pattern 2 } & \{1.0, & 0.5, & 0.25, & 0.0\} \\ \text { Pattern 3 } & \{1.0, & 0.1, & 0.05, & 0.0\}\end{array}$

The performance was evaluated in terms of the mean and standard deviation of the proportion difference in the prognostic factor (sex: male) between the comparison groups with the number of simulation iterations set at $R=1000$.

Mean of proportion difference $\bar{p}_{\text {diff }}: \bar{p}_{\text {diff }}=(1 / R) \sum_{r=1}^{R} p_{\text {diff }, r}$

Standard deviation of proportion difference $S D\left\{\bar{p}_{d i f f}\right\}: S D\left\{\bar{p}_{\text {diff }}\right\}=\sqrt{\{1 /(R-1)\} \sum_{r=1}^{R}\left(p_{\text {diff }, r}-\bar{p}_{\text {diff }, r}\right)^{2}}$,

$p_{W_{r}}$ : Proportion (\%) of wild type males at iteration $r$,

$p_{H r}$ : Proportion (\%) of homo type males at iteration $r$,

$p_{\text {diff }, r}=p_{W r}-p_{H r}$ : Proportion difference (\%) between wild type and homo type males at iteration $r$.

When all genotype groups reached their respective planned sample sizes, the simulation was terminated.

\section{Simulation Results}

The simulation results are shown in Figures 2 and 3 as contour line plots of the mean and standard deviation of the proportion difference in wild-type and homo-type males, with the probability (\%) of a male of the wild type on the $\mathrm{x}$-axis and the probability (\%) of a male of the homo type on the $y$-axis. Note that only $\left(p_{1}, p_{2}, p_{3}, p_{4}\right)=(1,0.1,0.05,0)$ of pattern 3 was shown in Figures 2 and 3 because the mean and standard deviation of the proportion difference are almost equivalent among the three registration probabilities. The number of non-registered subjects increased slightly as the registration probability got stricter.

Since the $p_{4}$ of all registration probabilities in this simulation were strict, i.e., $0 \%$, perhaps changing the other probabilities did not have a major impact on the mean proportion differences (\%). Under all of the conditions, the absolute values of the mean proportion difference in males became smaller than they were initially, which improved the comparability of the prognostic factor between the comparison groups.
The results according to the different conditions are described below. Regarding the planned sample size, the mean proportion difference between the comparison groups was almost unchanged, but the standard deviation of the proportion difference became smaller as the planned sample size became larger. In other words, although the improvement in the comparability of the comparison groups was, on average, equivalent regardless of the sample size, the larger the sample size became, the more stable the balance of the prognosis factor distribution that could be obtained by dynamic registration. Regarding the proportion of wild type, the mean and standard deviation of the proportion difference became smaller as the ratio of the wild type to the homo type became larger. In other words, the greater the number of candidates for registration, the greater the improvement in comparability between the two groups that can be obtained by dynamic registration.

\section{Discussion}

In observational studies, the distributions of prognostic factors might be unequal among comparison groups due to non-random allocation, and this imbalance degrades the reliability of the adjusted results as it increases. When the distribution of the prognostic factors hardly overlap among comparison groups, the interpretation of results from the conventional statistical analyses such as stratification, regression models, and propensity score methods is difficult. In this paper, we proposed a dynamic allocation method to improve the comparability between groups in observational studies. The proposed method was able to improve the comparability by dynamically deciding the registration of a candidate based on the background information of subjects already recruited and the candidate without any allocation. The dynamic allocation method is a design-based method to adjust for confounding factors. This method makes it easy to perform conventional methods of statistical analysis by enhancing the comparability before the data analyses. Although it was not considered in this study, it is possible to register, via dynamic registration, subjects who were initially not registered in randomized order, at a later point in time when a certain number of subjects have been reached.

The matched case-control approach is also a design-based method to adjust for confounding factors and may be better than the proposed method for optimizing comparability between groups. However, when conducting prospective cohort studies, we do not need to follow up nonregistered subjects since the proposed method judges the registration of subjects before starting follow-up of the subjects. Thus, one of the advantages of the proposed method may be that the resources and costs can be reduced as compared with following up of all subjects for the matching approach.

Our dynamic registration method requires the following conditions. First, since dynamic registration will result in non-registered subjects, non-registration of candidates should be acceptable in real practice and the number of candidates for registration should be larger than the planned sample size. According to the simulation results, a larger number of candidates for registration relative to the planned sample size allows for greater mitigating effect on imbalances in prognosis factor distribution. Also, a larger planned sample size allows for a more stable balance of prognosis factor distribution by dynamic registration. From the standpoint of restrictions and costs in real practice, we need to evaluate and determine in advance what percentage of nonregistered subjects is acceptable. Second, the prognostic factors and their levels including unexpected values for dynamic registration need to be determined in advance. In the case of a long-term study, 
Citation: Sugihara M, Morita S, Yamanouchi N, Sakai S, Ohba N, et al. (2012) Dynamic Registration Method with Balancing for Prognostic Factors in Observational Studies. J Biomet Biostat S7:012. doi:10.4172/2155-6180.S7-012

Page 6 of 6

stratification by time of accrual might be needed as a factor for the dynamic registration due to changes of the medical environment. Moreover, if medical treatments possibly related to the outcomes after registration are planned, these should be considered in the dynamic registration. The number of prognostic factors should be narrowed down as much as possible, because having as few prognostic factors as possible improves the comparability obtained by dynamic registration per prognostic factor. Third, a central registration system needs to be set up because the decision of whether to register a candidate must be made instantaneously. Moreover, the system needs to be coordinated so that registration conditions such as registration probabilities can be changed as needed in response to situations such as the registration of a subject who deviates substantially from the planned subject group. Lowering the registration probability can be expected to reduce any bias in prognosis factor distribution to a greater degree, but it increases the number of non-registered subjects and delays the registration of subjects. Therefore, we need to set appropriate registration conditions in consideration of how bias in prognostic factors between comparison groups will be allowed based on the registration situation and confirm the performance through simulation studies in advance.

The dynamic registration method may contribute to improvement of study quality as well as reduce resources and costs in the case of observational studies designed to compare unallocatable factors.

\section{Acknowledgments}

We thank the anonymous reviewers for helpful comments that have improved the presentation of this article.

\section{References}

1. McEntegart D (2003) The pursuit of balance using stratified and dynamic randomization techniques: an overview. Drug Inf J 37: 293-308.

2. Taves DR (1974) Minimization: a new method of assigning patients to treatment and control groups. Clin Pharmacol Ther 15: 443-453.

3. Pocock SJ, Simon R (1975) Sequential treatment assignment with balancing for prognostic factors in the controlled clinical trials. Biometrics 31: 103-115.

4. Scott NW, McPherson GC, Ramsay CR, Campbell MK (2002) The method of minimization for allocation to clinical trials. a review. Control Clin Trials 23: 662674.

5. Hagino A, Hamada C, Yoshimura I, Ohashi Y, Sakamoto J, et al. (2004) Statistical comparison of random allocation methods in cancer clinical trials. Control Clin Trials 25: 572-584.

6. Ando Y, Saka H, Ando M, Sawa T, Muro K, et al. (2000) Polymosphisms of UDP-glucuronosyltransferase gene and irinotecan toxicity: a pharmacogenetic analysis. Cancer Res 60: 6921-6926.

7. Innocenti F, Undevia SD, lyer L, Chen PX, Das S, et al. (2004) Genetic variants in the UDP-glucuronosyltransferase $1 \mathrm{~A} 1$ gene predict the risk of severe neutropenia of irinotecan. J Clin Oncol 22: 1382-1388.

8. D’Agostino RB Jr, D'Agostino RB Sr (2007) Estimating treatment effects using observational data. JAMA 297: 314-316.

9. Rosenbaum PR, Rubin DB (1983) The central role of the propensity score in observational studies for causal effects. Biometrika 70: 41-55.

10. Rubin DB (1997) Estimating causal effects from large data sets using propensity scores. Ann Intern Med 127: 757-763.

This article was originally published in a special issue, Medical statistics: Clinical and experimental research handled by Editor(s). Dr. Herbert Pang, Duke University, USA. 